\title{
Fluticasone induces apoptosis in peripheral T-lymphocytes: a comparison between asthmatic and normal subjects
}

\author{
M. Melis*, L. Siena*, E. Pace*, M. Gjomarkaj*, M. Profita*, A. Pirazzoli", M. Todaro", G. Stassi", \\ G. Bonsignore ${ }^{+}$, A.M. Vignola ${ }^{+}$
}

Fluticasone induces apoptosis in peripheral T-lymphocytes: a comparison between asthmatic and normal subjects. M. Melis, L. Siena, E. Pace, M. Gjomarkaj, M. Profita, A. Pirazzoli, M. Todaro, G. Stassi, G. Bonsignore, A.M. Vignola. (C)ERS Journals Ltd 2002.

ABSTRACT: Apoptosis is an important mechanism allowing inflammation to be limited. Glucocorticoids are the most effective anti-inflammatory agents in asthma therapy and induce cell apoptosis.

Since T-lymphocytes are critically involved in airway inflammation in asthma, the effects of fluticasone propionate (FP) on apoptosis in unstimulated and in interleukin (IL)-2 stimulated peripheral blood T-lymphocytes (PBTs) isolated from 14 normal and 19 mild-to-moderate asthmatic subjects were evaluated. Apoptosis was evaluated by: deoxyribonucleic acid (DNA) fragmentation electrophoresis, DNA content, annexin V binding, apoptosis related markers (Fas, B-cell lymphona leukaemia-2 (Bcl-2), Bax, and CD25), and by electron microscopy.

FP induced apoptosis in unstimulated PBTs of normal and asthmatic subjects in a time-dependent fashion. In asthma, this effect was associated with a significant decrease of Bcl-2 expression, and with an increase of Bax/Bcl-2 ratio. In PBTs of asthmatics, FP also reduced Fas and CD25 expression. Moreover, in IL-2-stimulated PBTs from both asthmatics and normal subjects, FP was able to induce apoptosis and to reduce $\mathrm{Bcl}-2$, Fas and CD25 expression, whereas negligible effects were detected on Bax expression.

This study shows that the glucocorticosteroid, fluticasone, increases apoptosis and modulates expression of apoptosis-related markers in unstimulated and in interleukin-2 stimulated T-lymphocytes. This points towards a potential mechanism by which fluticasone exerts its anti-inflammatory effects.

Eur Respir J 2002; 19: 257-266.
*Istituto di Fisiopatologia Respiratoria, Consiglio Nazionale delle Ricerche, Palermo, "Glaxo Wellcome, Verona, Dept of Surgical, Anatomical and Oncological Sciences Human Anatomy Section, Università degli Studi, Palermo, and Istituto di Medicina Generale e Pneumologia, Università degli Studi, Palermo, Italy.

Correspondence: M. Melis, Istituto di Fisiopatologia Respiratoria, Consiglio Nazionale delle Ricerche, Via Trabucco, 180-90146 Palermo, Italy.

Fax: 390916882165

E-mail: melis@ifr.pa.cnr.it

Keywords: Apoptosis asthma

fluticasone propionate inflammation

Received: April 232001

Accepted after revision September 5 2001
Asthma is a disease characterized by a chronic airway inflammation [1] in which T-lymphocytes play a central role [2]. In the airways of asthmatics T-lymphocytes are functionally activated and, by the release of a wide range of cytokines, regulate the recruitment of inflammatory cells, such as eosinophils [3]. In addition, most of the T-lymphocytes infiltrating the airways of asthmatics are not apoptotic, suggesting that the persistence of airway inflammation may also depend upon their increased survival in the bronchial mucosa [4]. In addition, in both acute severe and moderate asthma, peripheral T-lymphocytes are characterized by an increased activation state, as they express higher levels of CD25, human leukocyte antigen (HLA)-DR, and very late activation antigen (VLA)-1 than in normal subjects [5].

Apoptosis, a form of programmed cell death characterized by specific morphological features, is one of the mechanisms involved in the control of T-lymphocytes homeostasis [6, 7], causing the deletion of autoreactive $\mathrm{T}$ - and B-lymphocytes [8]. In this context, molecules belonging to the B-cell lymphoma leukaemia-2 (Bcl-2)/Bax system and to the Fas/ Fas-ligand system, play a crucial role in the regulation of the apoptotic process. In particular, Bcl-2 is an intracellular protein that inhibits apoptosis while Bax counteracts the anti-apoptotic function of $\mathrm{Bcl}-2$ by binding to this molecule [9]. Fas is a membrane protein that, when activated by its ligand, induces apoptosis $[10,11]$.

Fluticasone propionate (FP) is a glucocorticoid that exerts a potent anti-inflammatory activity and is commonly used in the treatment of asthma. FP inhibits human T-cell migration and proliferation in vitro and significantly reduces mast cells, eosinophils, and T-lymphocytes numbers in bronchoalveolar lavage [12]. Although the reduction of eosinophil numbers is also due to the induction of apoptosis [13], it is not known whether FP can exert a pro-apoptotic activity on T-lymphocytes as well.

The aims of the present study were: 1) to evaluate the effect of FP on apoptosis of both resting and interleukin (IL)-2 stimulated T-lymphocytes isolated from the peripheral blood of asthmatic patients; 2) to investigate the ability of FP to modulate T-lymphocyte expression of the related apoptotic markers Bcl-2, Bax and Fas, and of the cell activation marker CD25. 


\section{Materials and methods}

\section{Subjects}

Nineteen patients (median age and 25-75 percentiles: 44; 27-61 yrs) were selected according to the criteria of the American Thoracic Society. All patients were characterized by a reversible airway obstruction (forced expiratory volume in one second (FEV1) values expressed as median and 25-75 percentiles: 72 (51-99)) assessed by an increase of $15 \%$ of FEV1 after inhalation of $200 \mu \mathrm{g}$ of salbutamol. The severity of asthma was graded according to the AAs score [14]. Patients with persistent asthma were selected according to the World Health Organization/National Heart, Lung and Blood Institute (WHO/NHLBI) report and with an AAs [14] score of 2 ( $n=8$ patients) to 3 ( $\mathrm{n}=11$ patients).

Fourteen normal healthy subjects (age: 37; $28-$ 44 yrs) were enrolled. None of them had any previous history of lung or allergic disease. All subjects had a normal lung function test (FEV1: 104 (98-110)) and a negative allergy skin test. The enrolled patients did not undergo anti-inflammatory or steroid therapies during the two weeks prior to the study. All subjects were nonsmokers and had given their informed consent for the procedure. This study fulfilled the criteria of the Ethics Committee of the Hospital.

\section{Isolation of peripheral blood T-lymphocytes}

Peripheral blood T-lymphocytes (PBTs) were obtained from $40 \mathrm{~mL}$ of heparinized blood from asthmatics and normal donors as described previously [15]. The recovered PBT fraction was $>97 \%$ CD3+ as assessed by cytofluorimetric analysis.

\section{T-lymphocytes cultures}

PBTs were incubated with or without recombinant interleukin (IL)-2 (Genzyme, Cambridge, MA, USA) $\left(1,000 \mathrm{UI} \cdot \mathrm{mL}^{-1}\right)$ and with or without FP (Glaxo Wellcome, Verona, Italy) at a final concentration of $10^{-7} \mathrm{M}$. This concentration was used because, in preliminary dose-response curve experiments (FP from $1 \times 10^{-10}-1 \times 10^{-4} \mathrm{M}$ ), it had been demonstrated that $1 \times 10^{-7} \mathrm{M}$ is the optimal concentration able to induce cell apoptosis. Moreover, this concentration is within the pharmacological range [16]. PBTs were cultured $\left(37^{\circ} \mathrm{C}, 5 \% \mathrm{CO}_{2}\right)$ for $24,48,72$, and $144 \mathrm{~h}$, respectively, and cell apoptosis was evaluated as described below.

\section{Annexin $V$ binding}

Apoptosis of stimulated and unstimulated PBTs was determined by using a FACStar Plus analyser (Becton Dickinson, San Jose, CA, USA) equipped with an Argon-Ion Laser (Coherent Innova 70, Becton Dickinson) and Consort 32 computer support, by evaluating Annexin V expression using a commercial kit (Bender MedSystem, Vienna, Austria), following the manufacturer's directions. The Annexin $\mathrm{V}$ positive/propidium iodide (PI) negative cells were considered apoptotic cells. During apoptosis the cells became reactive with Annexin V after the onset of chromatin condensation but prior to the loss of the plasma membrane's ability to exclude PI. Therefore, by staining cells with a combination of fluoresceinated Annexin V and PI, it is possible to detect early apoptotic cells (Annexin V Positive-PI negative) and to differentiate them from necrotic cells (PI positive).

\section{Deoxyribonucleic acid gel electrophoresis}

Deoxyribonucleic acid (DNA) fragmentation into nucleosomal bands was detected by agarose gel electrophoresis, as previously described [17].

\section{Deoxyribonucleic acid content}

PBTs were washed twice in phosphate buffered saline (PBS), resuspended at a concentration of $1 \times 10^{6}$ cells $\cdot \mathrm{mL}^{-1}$ and fixed in $70 \%$ cold ethanol for $30 \mathrm{~min}$ at $4^{\circ} \mathrm{C}$. The cells were washed twice in PBS and incubated in sodium citrate buffer $(0.1 \%)$ containing $0.1 \%$ Triton $\mathrm{X}-100,50 \mu \mathrm{g} \cdot \mathrm{mL}^{-1}$ ribonuclease and $50 \mu \mathrm{g} \cdot \mathrm{mL}^{-1}$ PI for $30 \mathrm{~min}$ at room temperature in the dark. PI fluorescence of individual cells was analysed by FACStar Plus cytometer (Becton Dickinson) and data were expressed in a log scale.

\section{Electron microscopy}

PBTs were centrifuged, washed twice in PBS and resuspended in fixative universal solution $(4 \%$ paraformaldehyde and $1 \%$ glutaraldehyde in $0.1 \mathrm{M}$ phosphate buffer) for $1 \mathrm{~h}$. Cells were then washed in PBS, postfixed in $2 \%$ osmium tetroxide for $1 \mathrm{~h}$ and rinsed twice in PBS, followed by dehydration through a series of alcohols (30-100\%, 5-min steps). Embedding was performed in epoxy resin (Epon812). Polymerization was carried out at $60^{\circ} \mathrm{C}$ for $48 \mathrm{~h}$. Semi-thin sections $(0.5 \mu \mathrm{m})$, cut using a ultramicrotome (Ultracut-E, Reichert-Yung, Jena, Germany), were mounted onto slides and warm-stained with methylene blue. Screening and photography were performed using a light microscope (Zeiss, Jena, Germany). Ultra-thin sections $(80 \mathrm{~nm})$ were mounted onto nickel grids, and counter-stained with $8 \%$ uranyl acetate in methanol for $10 \mathrm{~min}$ and lead citrate for 1-2 min. Cells were analysed by a 1220 electron microscope (JEOL, Tokyo, Japan).

\section{Determination of apoptotic markers}

The expressions of Fas, Bcl-2, Bax, and CD25 were evaluated using the same experimental conditions as described earlier. To evaluate intracellular expression of Bcl-2 and Bax proteins, cells were permeabilized using a commercial fix-perm cell permeabilization kit (Caltag Laboratories, Burlingame, CA, USA) following the manufacturer's directions. Cells were 
then washed, resuspended in PBS at a concentration of $5 \times 10^{6} \mathrm{~mL}^{-1}$ and $0.1 \mathrm{~mL}$ aliquots were incubated in the dark $\left(30 \mathrm{~min}, 4^{\circ} \mathrm{C}\right)$ with a flouorescin isothiocyanate (FITC)-conjugated mouse anti-Bcl-2 clone 124, (Dakopatts, Glostrup, Denmark) or with the antiBax monoclonal antibody (MoAb) (unconjugated Bax p19 rabbit antihuman IgG1, Santa Cruz Biotechnology, Santa Cruz, CA, USA) followed by a phycoerythrin (PE)-conjugated antirabbit immunoglobulin (Ig)-G1 (Sigma, St Louis, MO, USA). For indirect immunofluorescence, an irrelevant unconjugated IgG1 (X-931, Dakopatts) followed by PE-conjugated rabbit antimouse IgG (Sigma) was used as a negative control. CD25 and Fas expression were evaluated by using antihuman (PE)-conjugated CD25 clone, ACT-1, (Dakopatts), and antihuman PE-conjugated CD95 clone DX2 (Dakopatts) MoAbs, respectively. PE-conjugated (X-928, Dakopatts) and FITC-conjugated mouse antihuman IgG1 (X-927, Dakopatts) were used as negative controls for direct immunofluorescence experiments, while PE-conjugated mouse antirabbit IgG (Dakopatts) was used as a negative control for indirect immunofluorescence experiments. Cells $\left(1 \times 10^{4}\right)$ were evaluated. The expression of Fas and CD25 are presented as the percentage of positive cells; results of intracellular expression of Bcl-2 and Bax proteins are expressed as mean peak channel fluorescence [18].
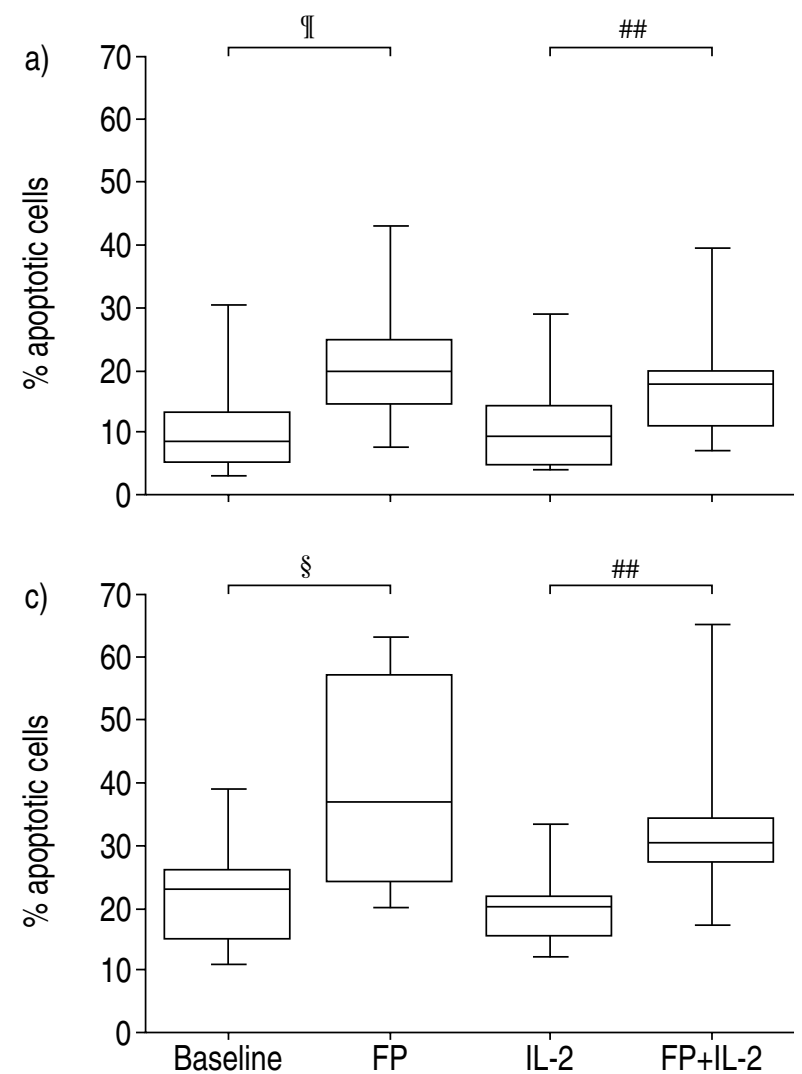

\section{Statistical analysis}

Data are expressed as medians and 25-75 percentiles. The Mann-Whitney U-test was used for unpaired comparisons. For multiple correlations, analysis for statistical significance was performed using a one-way analysis of variance (ANOVA test). For all tests $\mathrm{p}<0.05$ was accepted as the threshold of statistical significance.

\section{Results}

Induction of apoptosis by fluticasone propionate on peripheral blood T-lymphocytes

Spontaneous apoptosis of PBTs isolated both from asthmatic and normal subjects was evident after 72 h (asthma: $\mathrm{p}<0.002$ (ANOVA); normal subjects: $\mathrm{p}<0.003$, (ANOVA)) and smoothly increased after $144 \mathrm{~h}$ (fig. 1) (asthma: $\mathrm{p}<0.002$; normal subjects: $\mathrm{p}<0.002)$. Negligible percentages of apoptotic cells were detected after 24 and $48 \mathrm{~h}$ of incubation in both study groups (data not shown). To verify whether the presence of FP was able to increase the number of apoptotic cells, PBT was incubated with FP at a molar concentration comparable to its in vivo pharmacological range (see Materials and methods). The

b)

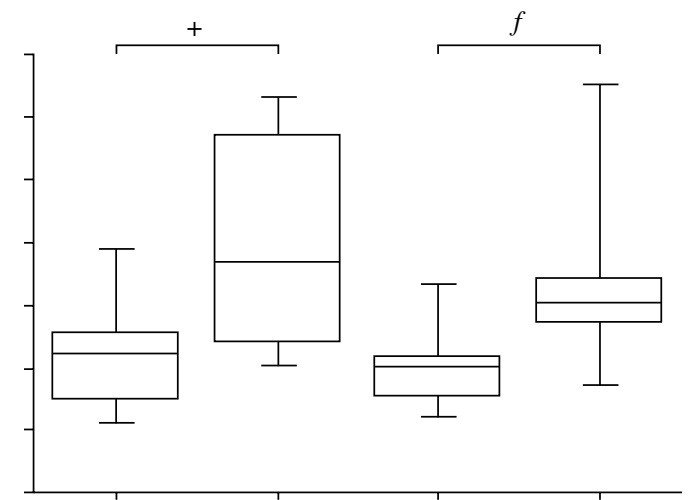

d)

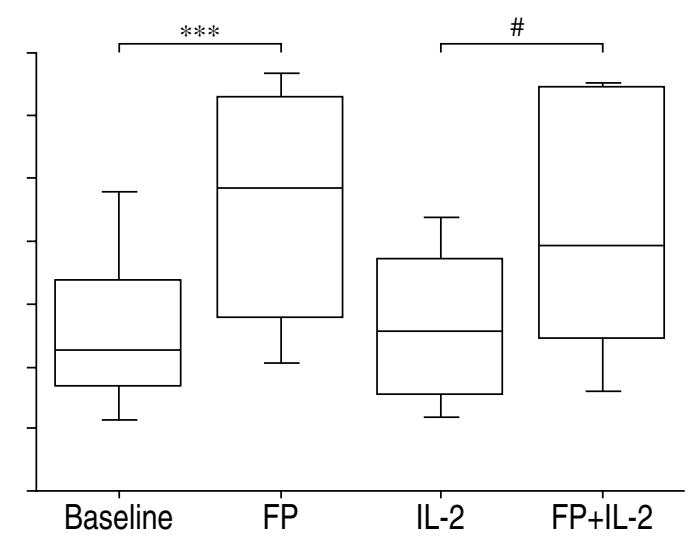

Fig. 1.-Apoptosis of peripheral blood T-lymphocytes (PBTs) isolated from normal (a: $72 \mathrm{~h}, \mathrm{~b}$ : $144 \mathrm{~h}$; n=14) and asthmatic (c: $72 \mathrm{~h}$, $\mathrm{d}: 144 \mathrm{~h} ; \mathrm{n}=19)$ subjects. PBTs were isolated as described in the Materials and methods section and apoptosis was quantified using a fluorescein isothiocyanate-conjugated Annexin V simultaneously with dye exclusion of propidium iodide (PI) on PBTs cultured in different experimental conditions. Data are expressed as \% of Annexin positive-PI negative cells (mean \pm SD). Statistical analysis was by ANOVA test. FP: fluticasone propionate; IL-2: interleukin-2. ${ }^{\#}: \mathrm{p}<0.04 ;{ }^{\uparrow}: \mathrm{p}<0.02 ;{ }^{+}: \mathrm{p}<0.007 ;{ }^{\S}: \mathrm{p}<0.004 ;{ }^{* * *}: \mathrm{p}<0.001 ;{ }^{f}: \mathrm{p}<0.0006{ }^{\# \#}: \mathrm{p}<0.0005$. 
incubation with FP strongly increased the percentages of apoptotic cells in both study groups in a timedependent manner (normal subjects: $72 \mathrm{~h}$ medium versus $\mathrm{FP} p<0.02 ; 144 \mathrm{~h}$ medium versus $\mathrm{FP} p<0.004$; asthmatic subjects: $72 \mathrm{~h}$ medium versus $\mathrm{FP} p<0.007$; $144 \mathrm{~h}$ medium versus FP $\mathrm{p}<0.001$ ) (fig. 1). No significant differences were detected between asthmatics and normal subjects.

Since the presence of IL-2 has been demonstrated to rescue T-lymphocytes from apoptosis [19], the role played by FP in affecting the apoptotic phenomenon in a IL-2-stimulated model was investigated. As expected, the incubation with IL-2 decreased the percentages of apoptotic PBTs in both asthmatics and normal subjects, rescuing PBTs from spontaneous apoptosis $(\mathrm{p}<0.05$; with the exception of asthmatics after $144 \mathrm{~h}$ of incubation (nonsignificant)). Interestingly, the incubation with FP counteracted the rescue activity of IL-2, causing a significant increase in the percentages of apoptotic IL-2-treated PBTs in both study groups (figs. 1a and $1 \mathrm{~b}$; normal subjects, $72 \mathrm{~h}$ IL-2 versus FP+IL-2, $\mathrm{p}<0.0006 ; 144 \mathrm{~h}$ IL-2 versus $\mathrm{FP}+\mathrm{IL}-2, \mathrm{p}<0.0005$; asthmatic subjects, $72 \mathrm{~h}$ IL-2 versus $\mathrm{FP}+\mathrm{IL}-2, \mathrm{p}<0.0006 ; 144 \mathrm{~h}$ IL-2 versus
FP+IL-2, $\mathrm{p}<0.04)$. The evaluation of DNA content demonstrated that FP exerted pro-apoptotic, in addition to, necrotic effects on PBTs (fig. 2a). After FP exposure, DNA damage was observed (fig. 2b; lanes 4 and 6) but DNA fragmentation was not typical, probably due to the presence of necrotic cells, which obscured the evidence of DNA laddering. In addition, the use of electron microscopy revealed, in FP-incubated PBTs, the presence of typical morphological changes due to apoptosis (fig. 3).

\section{Effect of fluticasone propionate on B-cell lymphoma leukaemia-2 and Bax markers expression by peripheral blood T-lymphocytes}

Similar levels of Bcl-2 were expressed by PBTs freshly isolated from both asthmatic patients and normal subjects (fig. 4). Bcl-2 expression in normal subjects spontaneously decreased in a time-dependent fashion. In contrast, in asthmatic patients, Bcl-2 expression remained stable at all time-points, reaching a statistical significance at $144 \mathrm{~h} \quad(\mathrm{p}<0.006)$. The incubation with FP significantly reduced the a)

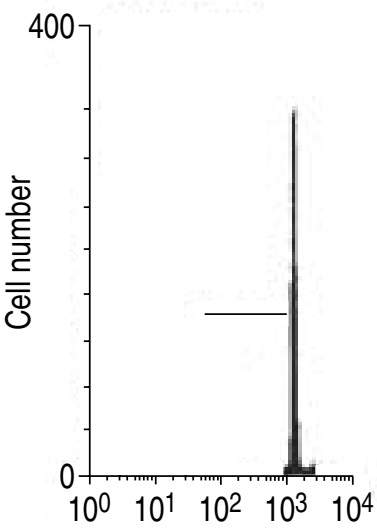

b)
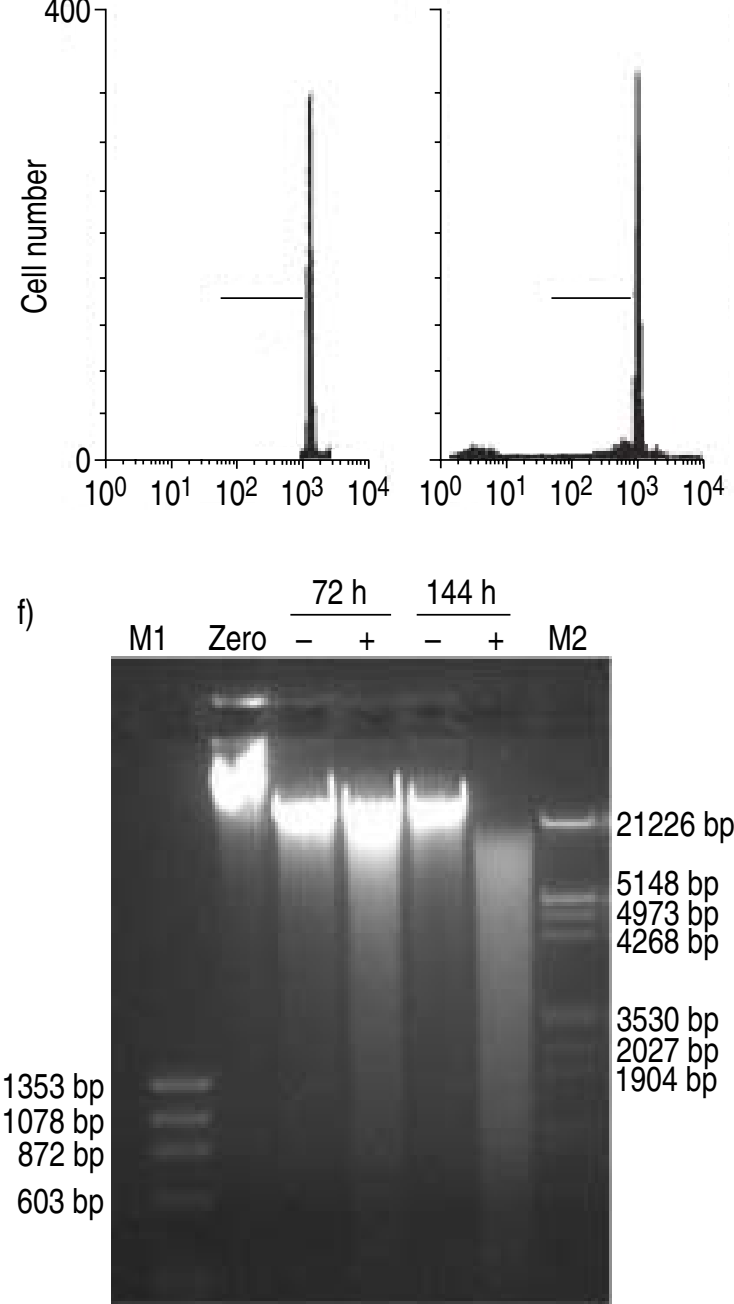

c)

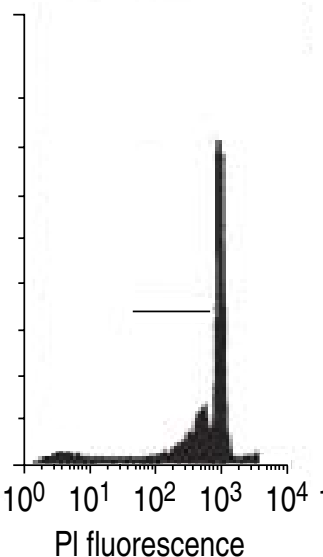

d)

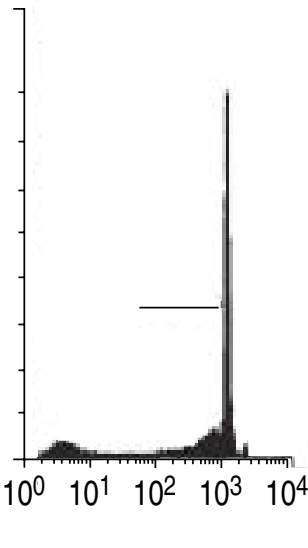

e)

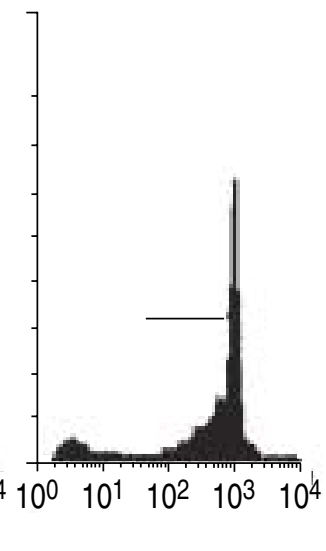

Fig. 2.-Effect of fluticasone propionate (FP) on deoxyribonucleic acid (DNA) fragmentation and hypodiploid DNA content. DNA fragmentation and hypodiploid DNA content were evaluated on peripheral blood T-lymphocytes (PBTs) from asthmatic patients cultured with FP $\left(1 \times 10^{-7} \mathrm{M}\right)$ for 72 and $144 \mathrm{~h}$. a)-e) Representative cytofluorimetric analysis of hypodiploid DNA content after staining with propidium iodide (PI) (a): $0 \mathrm{~h}$ baseline; b): $72 \mathrm{~h}$ baseline; c) $72 \mathrm{~h} \mathrm{FP}$; d) $144 \mathrm{~h}$ baseline; e) $144 \mathrm{~h} \mathrm{FP}$ ). f) Agarose gel electrophoresis of DNA. Lane M1: $\phi x 174$ molecular weight marker; lane zero: DNA from freshly-isolated PBTs; lane 3: DNA isolated from PBTs cultured in medium in the absence of FP for $72 \mathrm{~h}$; lane 4: DNA isolated from PBTs cultured with FP for $72 \mathrm{~h}$; lane 5: DNA isolated from PBTs cultured in medium in the absence of FP for $144 \mathrm{~h}$; lane 6: DNA isolated from PBTs cultured with FP for $144 \mathrm{~h}$; lane M2 represents $\lambda$ DNA Eco RI Hind III molecular weight marker. 

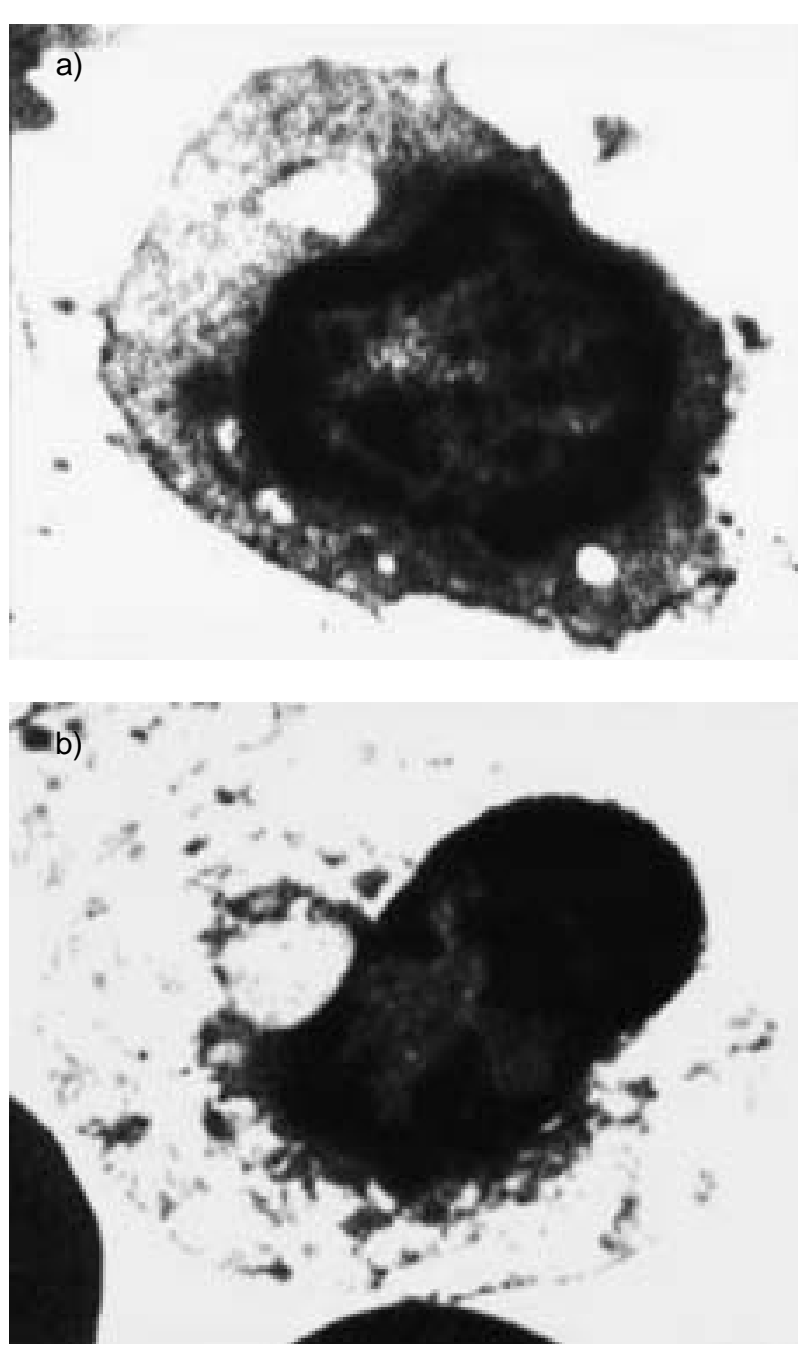

Fig. 3.-Ultrastructural level of morphological changes observed in peripheral blood T-lymphocytes (PBTs) following fluticasone propionate exposure. a) Early stage of apoptosis, characterized by cytoplasmic boiling and chromatin condensation. b) Late stage of apoptosis characterized by collapsed chromatin down into crescents along the nuclear envelope and, in the cytoplasm, dilation of cisternae.

expression of $\mathrm{Bcl}-2$ on PBTs from asthmatic patients (72 h: medium versus FP, $\mathrm{p}<0.04 ; 144 \mathrm{~h}$ : medium versus $\mathrm{FP}, \mathrm{p}<0.05)$, but not on PBTs from normal subjects (fig. 4).

The incubation with IL-2 was evaluated next, to determine whether it was able to modify the expression of Bcl-2 by PBTs, and whether FP was able to counteract the effect of IL-2 on the expression of this marker. IL-2 significantly increased the expression of Bcl-2 on PBTs isolated from both normal and asthmatic subjects at all time-points (normal subjects, $72 \mathrm{~h}: \mathrm{p}<0.02 ; 144 \mathrm{~h}: \mathrm{p}<0.003$; asthmatic subjects, $72 \mathrm{~h}: \mathrm{p}<0.0005$; $144 \mathrm{~h}: \mathrm{p}<0.05$ ) (fig. 4) and FP was able to counteract this effect by significantly decreasing the expression of $\mathrm{Bcl}-2$ both in normal and asthmatic subjects (normal subjects, $72 \mathrm{~h}: \mathrm{p}<0.05$; $144 \mathrm{~h}: \mathrm{p}<0.04$; asthmatic subjects, $72 \mathrm{~h}: \mathrm{p}<0.03$; 144 h: $\mathrm{p}<0.02$ ) (fig. 4).

The authors further investigated whether the incubation with FP was able to affect the expression of Bax molecule on unstimulated as well as on IL-2-stimulated PBTs, isolated from both asthmatics and normal subjects. The incubation with FP had no effect on Bax expression by PBTs isolated both from asthmatics and normal subjects while, as expected, IL-2 significantly decreased the expression of this molecule on PBTs isolated from the two study groups at all time points. Interestingly, the addition of FP was able to restore the expression of Bax molecule on IL-2-stimulated PBTs. (fig. 5).

The effects of $\mathrm{FP}$ on $\mathrm{Bax} / \mathrm{Bcl}-2$ ratio were also examined. Although the incubation with FP increased the $\mathrm{Bax} / \mathrm{Bcl}-2$ ratio both in PBTs from normal subjects and asthmatics, these effects were statistically significant only in asthmatics after $144 \mathrm{~h}$ in unstimulated PBTs and after 72 and $144 \mathrm{~h}$ in IL-2-stimulated PBTs (table 1).

Effect of fluticasone propionate on Fas expression by peripheral blood T-lymphocytes

The number of Fas positive cells was higher in the PBT population freshly isolated from asthmatic patients than in the PBT population freshly isolated from normal subjects $(\mathrm{p}<0.006$, Mann-Whitney U-test) and this difference was still present after $72 \mathrm{~h}$ of incubation $(\mathrm{p}<0.04)$. The incubation with FP was able to significantly decrease the percentage of Fas positive PBTs in asthmatics $(p<0.03)$, but not in normal subjects after $72 \mathrm{~h}$, while no effect was detected after $144 \mathrm{~h}$ of incubation (fig. 6).

The incubation of PBTs with IL-2 significantly increased Fas expression on these cells in both normal subjects (72 h: $\mathrm{p}<0.002 ; 144 \mathrm{~h}: \mathrm{p}<0.0008)$ and asthmatic patients (72 h: $p<0.007 ; 144 \mathrm{~h}: \mathrm{p}<0.0008)$. The incubation with FP counteracted the effect of IL-2 on PBTs significantly reducing the Fas expression both in normals $(72 \mathrm{~h}: \mathrm{p}<0.008 ; 144 \mathrm{~h}: \mathrm{p}<0.0006)$ and asthmatic (72 h: $\mathrm{p}<0.004 ; 144 \mathrm{~h}: \mathrm{p}<0.0006)$ samples (fig. 6).

Effect of fluticasone propionate on CD25 expression by peripheral blood T-lymphocytes

The numbers of CD25 positive cells were higher in the PBT population freshly isolated from asthmatics than in the PBT population freshly isolated from normal subjects, and this difference was still present after 72 and $144 \mathrm{~h}$ of incubation $(\mathrm{p}<0.04$ and $\mathrm{p}<0.006$, respectively, Mann-Whitney U-test). FP significantly decreased the percentage of CD25 positive PBTs in both normal subjects (72 h: $\mathrm{p}<0.02 ; 144 \mathrm{~h}: \mathrm{p}<0.001)$ and asthmatics at all time points $(72 \mathrm{~h}: \mathrm{p}<0.005$; 144 h: $p<0.005$ ) (fig. 7). Moreover, IL-2 at all time points significantly increased the expression of this marker on PBTs isolated both from normal $(72 \mathrm{~h}$ : $\mathrm{p}<0.007 ; 144 \mathrm{~h}: \mathrm{p}<0.009)$ and asthmatic subjects (72 h: $\mathrm{p}<0.005 ; 144 \mathrm{~h}: \mathrm{p}<0.004)$ (fig. 7). Finally, the authors evaluated whether FP was able to counteract the effect of IL-2 on the CD25 expression by PBTs. The incubation with FP significantly reduced the 
a)

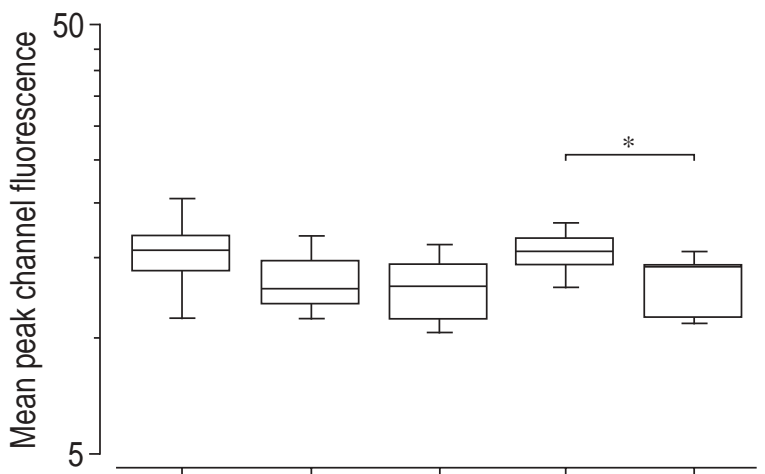

c)

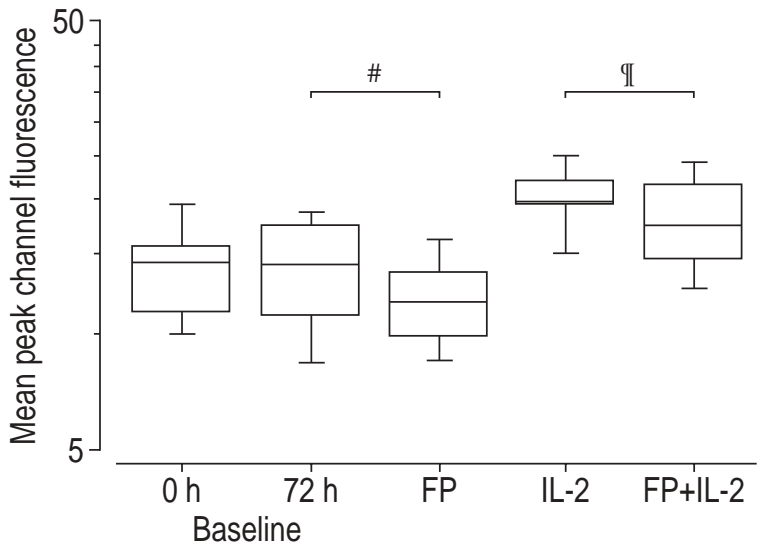

b)

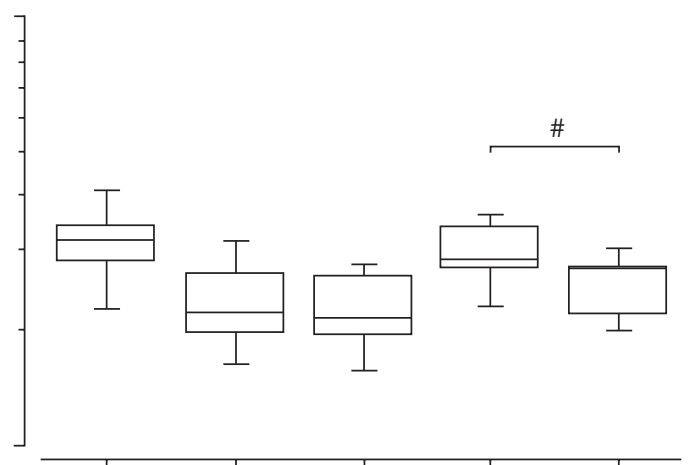

d)

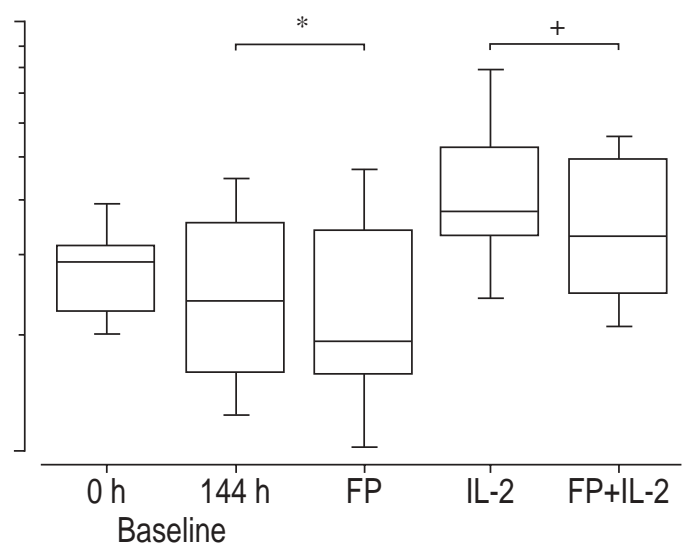

Fig. 4. - Expression of B-cell lymphoma leukaemia-2 (Bcl-2) by peripheral blood T-lymphocytes (PBTs) isolated from normal (a: 72 h, b: $144 \mathrm{~h} ; \mathrm{n}=10)$ and asthmatic (c: $72 \mathrm{~h}, \mathrm{~d}: 144 \mathrm{~h} ; \mathrm{n}=10)$ subjects. PBTs were isolated as described in "Materials and methods" and Bcl-2 expression was evaluated by using an anti-Bcl-2 monoclonal antibody on PBTs cultured in medium alone, in the presence of fluticasone propionate (FP), in the presence of interleukin-2 (IL-2), and in the presence of both FP and IL-2. Data are expressed as mean peak channel fluorescence $\left(\right.$ mean \pm SD). Statistical analysis was by analysis of variance (ANOVA) test. ${ }^{*}: \mathrm{p}<0.05 ;{ }^{\#}: \mathrm{p}<0.04 ;{ }^{\bullet}: \mathrm{p}<0.03{ }^{+}{ }^{*}: \mathrm{p}<0.02$.

expression of CD25 by IL-2-stimulated PBTs from asthmatics (72 h: $\mathrm{p}<0.05 ; 144 \mathrm{~h}: \mathrm{p}<0.005)$ but not from normal subjects (fig. 7 ).

\section{Discussion}

This study shows that FP induces apoptosis in unstimulated PBTs of normal and asthmatic subjects in a time-dependent manner. In addition, in asthma, this effect was associated with a significant decrease of Bcl-2 expression, and with an increase of the Bax/Bcl-2 ratio. In PBTs of asthmatics, FP also reduced Fas and CD25 expression. Moreover, in IL-2-stimulated PBTs from both asthmatics and normal subjects, FP was able to induce apoptosis and to reduce Bcl-2, Fas and CD25 expression, while negligible effects were detected on Bax expression.

T-lymphocytes in asthma release a large spectrum of inflammatory cytokines, which play a crucial role in the development of airway inflammation [3, 4]. Consequently, T-lymphocytes represent an important target of anti-inflammatory drugs, and particularly of steroids. FP is a glucocorticoid with a potent anti-inflammatory activity characterized by high affinity for the steroid binding site on the human lung glucocorticoid receptor (GR) [17]. It has been demonstrated that FP is more potent than other steroids in inhibiting in vitro human $\mathrm{T}$-cell migration and proliferation [17]. In addition, in asthmatics, FP treatment is able to significantly decrease the number of T-lymphocytes in bronchoalveolar lavage and in nasal mucosa $[12,20]$. Although this evidence shows that FP can decrease the number of T-lymphocytes, the mechanisms underlying this phenomenon have not been completely elucidated. Apoptosis plays a fundamental role in the control of the "tissue load" of cells at inflamed sites, and tends to limit inflammatory tissue injury and to promote resolution rather than progression of inflammation [21, 22]. Therefore, in the present study the authors evaluated whether the anti-inflammatory properties of FP may also be related to the induction of T-lymphocyte apoptosis.

The study first demonstrated that unstimulated in vitro cultured PBTs from asthmatic and normal subjects undergo spontaneous apoptosis in the same manner, and that the effect of FP on apoptosis of PBTs from mild-to-moderate asthmatics and normal subjects is similar (fig. 1). The data confirm previous observations [23], showing that long-term cultured T-lymphocytes undergo apoptosis spontaneously and are responsive to the pro-apoptotic effect of steroids. In this regard, it has been shown that dexamethasone and prednisone can induce apoptosis of normal 
a)

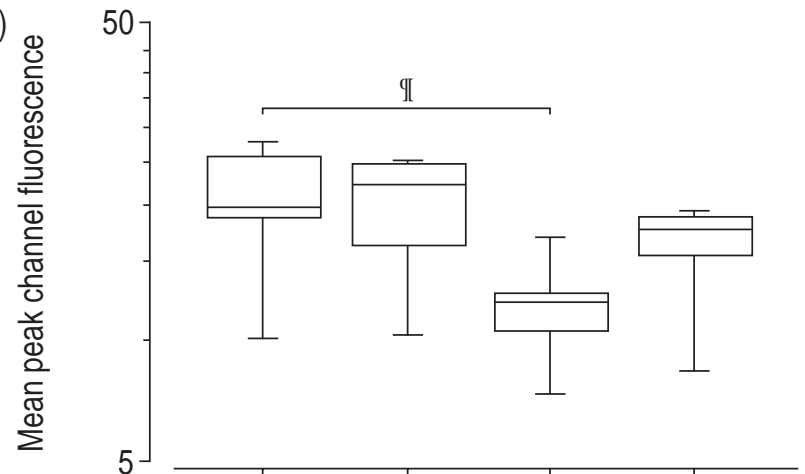

c)

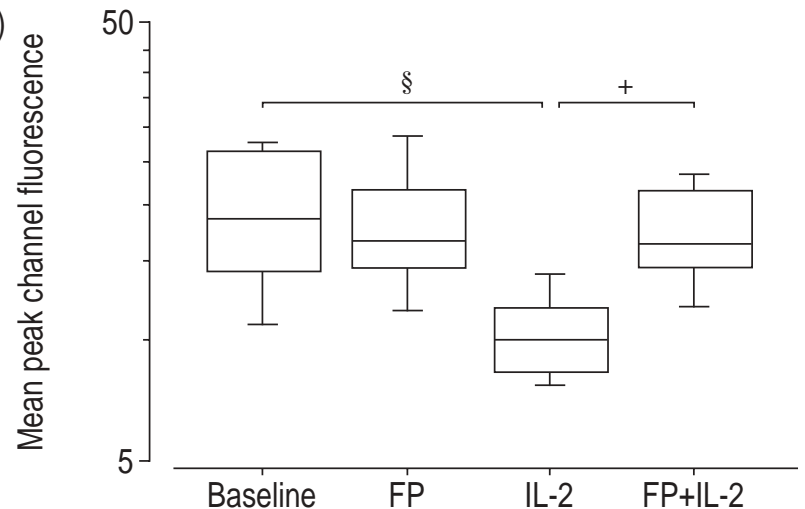

b)

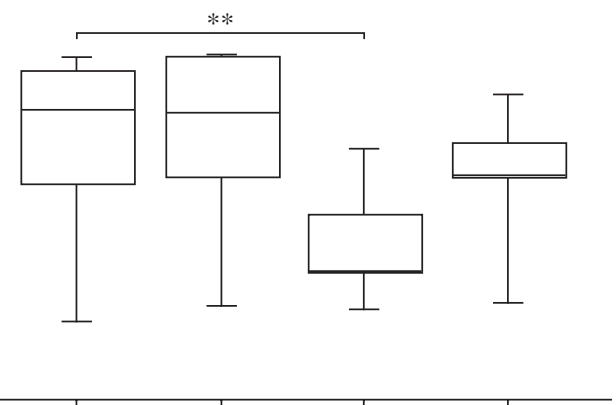

d)

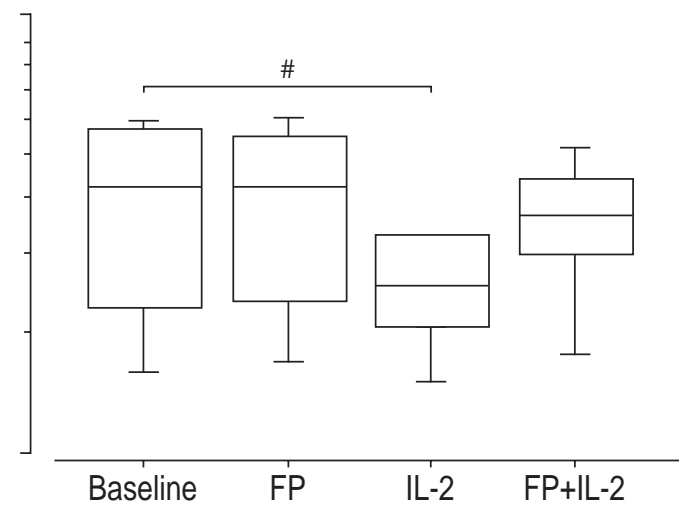

Fig. 5. - Expression of Bax by peripheral blood T-lymphocytes (PBTs) isolated from normal (a: $72 \mathrm{~h}, \mathrm{~b}$ : $144 \mathrm{~h}$; $\mathrm{n}=10$ ) and asthmatic (c: $72 \mathrm{~h}, \mathrm{~d}: 144 \mathrm{~h} ; \mathrm{n}=10)$ subjects. PBTs were isolated as described in "Materials and methods" and Bax expression was evaluated by using an anti-Bax monoclonal antibody on PBTs cultured in medium alone, in the presence of fluticasone propionate (FP), in the presence of interleukin-2 (IL-2), and in the presence of both FP and IL-2 . Data are expressed as mean peak channel fluorescence (mean \pm SD). Statistical analysis was by analysis of variance (ANOVA) test. ${ }^{\#}: \mathrm{p}<0.04 ;{ }^{\uparrow}: \mathrm{p}<0.02 ;{ }^{* *}: \mathrm{p}<0.01 ;{ }^{+}: \mathrm{p}<0.005 ;{ }^{\S}: \mathrm{p}<0.002$.

PBTs in a dose- and time-dependent manner [24, 25]. Accordingly, this study demonstrates that FP exerts the maximal in vitro proapoptotic effect, in both study groups, at a molar concentration $\left(1 \times 10^{-7}\right)$ that is comparable to its in vivo pharmacological range, and in a time-dependent manner.

To identify the mechanisms by which FP induces apoptosis on unstimulated in vitro cultured PBTs, the expression of the apoptosis related markers Bax and Bcl-2 was evaluated. It was found that FP decreased Bcl-2 expression in asthmatic but not in normal PBTs (fig. 4), suggesting that, in asthmatics, FP-induced apoptosis is partly mediated by the downregulation of the "survival" Bcl-2 molecule. In addition, the finding that Bcl-2 expression spontaneously decreases after 72 and $144 \mathrm{~h}$ of incubation in normal subjects, while the expression of this molecule remains stable in asthmatics (fig. 4), supports the hypothesis that the mechanisms leading to the regulation of the $\mathrm{Bcl}-2$ expression are altered in asthma, a phenomenon that is likely to be associated with the persistent inflammatory process characterizing the disease. Therefore, it is probable that FP is active in PBTs from asthmatics because it is able, at least partly, to restore the regulatory activity of these mechanisms.

The inhibitory action of FP on the biological

Table 1.-Bax/B-cell lymphoma leukaemia-2 ratio

\begin{tabular}{|c|c|c|c|c|c|c|}
\hline & \multicolumn{3}{|c|}{ Unstimulated PBT } & \multicolumn{3}{|c|}{ IL-2 stimulated PBT } \\
\hline & Baseline & FP & p-value & IL-2 & $\mathrm{FP}+\mathrm{IL}-2$ & p-value \\
\hline \multicolumn{7}{|l|}{ Normals } \\
\hline $72 \mathrm{~h}$ & $1.7(1.2-1.8)$ & $1.6(1.3-2.3)$ & NS & $0.7(0.6-0.8)$ & $1.2(1.1-1.4)$ & NS \\
\hline $144 \mathrm{~h}$ & $2(1.4-2.5)$ & $2.1(1.5-2.9)$ & NS & $0.8(0.7-1)$ & $1.2(1-1.5)$ & NS \\
\hline \multicolumn{7}{|l|}{ Asthmatics } \\
\hline $72 \mathrm{~h}$ & $1.6(0.9-2)$ & $1.8(1.1-2.6)$ & NS & $0.5(0.4-0.5)$ & $1(0.9-1.2)$ & $<0.04$ \\
\hline $144 \mathrm{~h}$ & $1.1(1-1.8)$ & $1.9(1.3-3.7)$ & $<0.01$ & $0.6(0.4-0.6)$ & $1.2(0.7-1.7)$ & $<0.007$ \\
\hline
\end{tabular}

Data are presented as median (25-75 percentiles) unless where otherwise indicated. FP: fluticasone propionate; IL-2: interleukin-2; PBT: peripheral blood T-lymphocytes; NS: nonsignificant. Statistical analysis by ANOVA. Data were considered statistically significant when $\mathrm{p}<0.05$. 

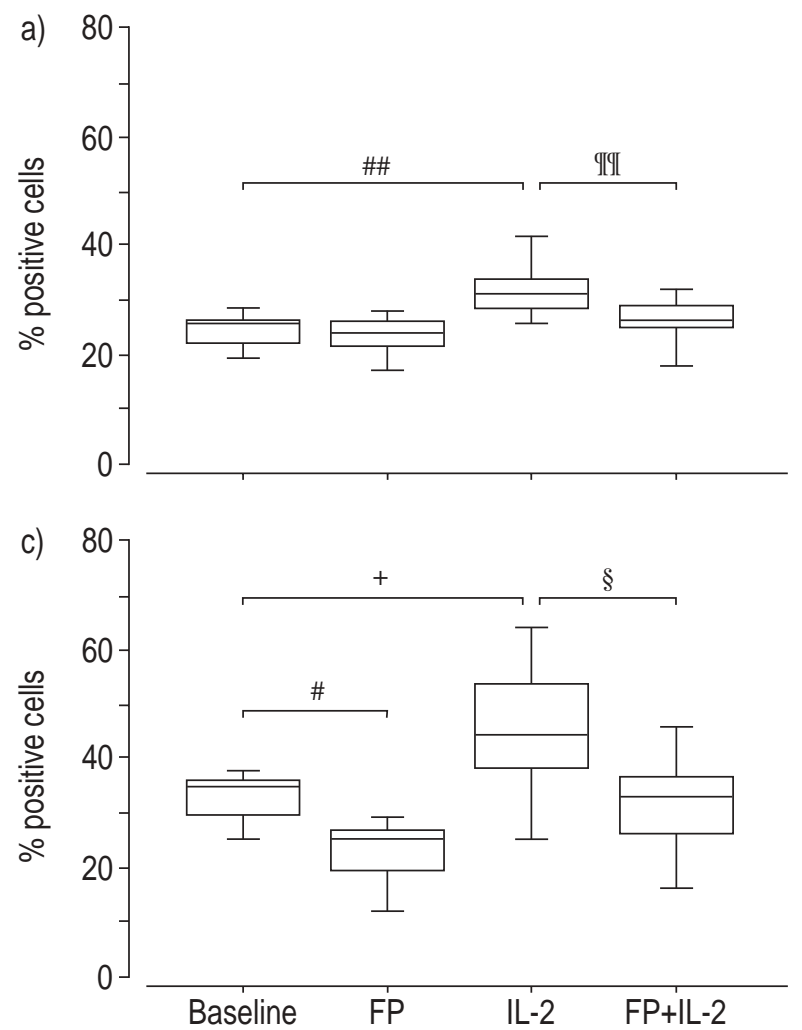

b)

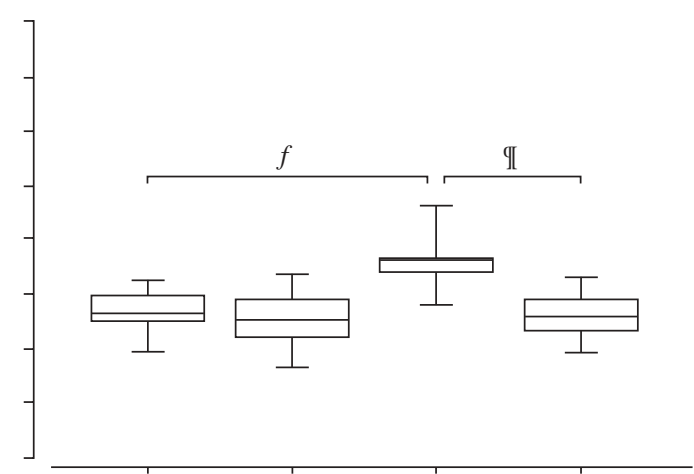

d)

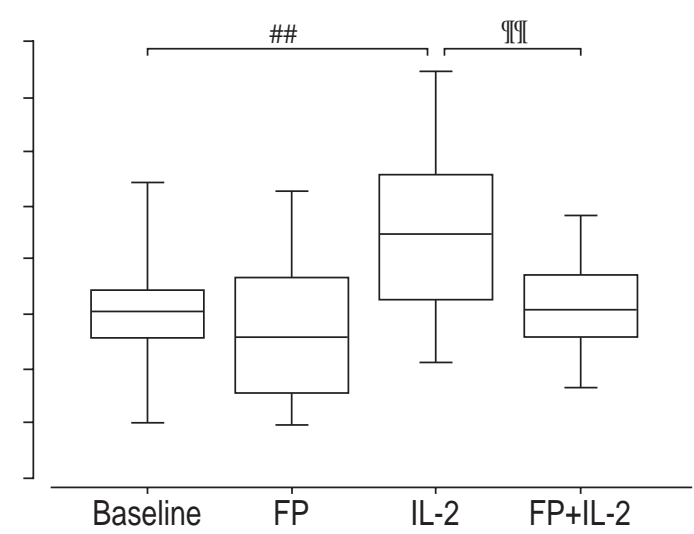

Fig. 6. - Expression of Fas receptor by peripheral blood T-lymphocytes (PBTs) isolated from normal (a: $72 \mathrm{~h}$, b: 144 h; n=10) and asthmatic (c: 72 h, d: $144 \mathrm{~h} ; \mathrm{n}=10$ ) subjects. PBTs were isolated as described in the Materials and methods section and Fas expression was evaluated by using an anti-Fas monoclonal antibody on PBTs cultured in medium alone, in the presence of fluticasone propionate (FP), in the presence of interleukin-2 (IL-2), and in the presence of both FP and IL-2. Data are expressed as \% of positive cells (mean \pm SD). Statistical analysis was by ANOVA test. ${ }^{\#}: \mathrm{p}<0.03 ;{ }^{\circledR}: \mathrm{p}<0.008 ;{ }^{+}: \mathrm{p}<0.007 ;{ }^{\S}: \mathrm{p}<0.004 ;{ }^{f}: \mathrm{p}<0.002 ;{ }^{\# \#}: \mathrm{p}<0.0008 ;{ }^{\natural}: \mathrm{p}<0.0006$.

activities of IL-2 represents another important mechanism by which this drug exerts its antiinflammatory and proapoptotic effects. It is widely accepted that activated T-lymphocytes require the continuous presence of IL-2 for clonal expansion and the prevention of apoptosis by upregulating the expression of Bcl-2. Incubation with steroids decreases IL-2 production by activated T-lymphocytes in vitro, and leads to the reduction of Bcl-2 expression and apoptosis. Conversely, the addition of IL-2 to the same cultures reinduces the expression of $\mathrm{Bcl}-2$ and prevents apoptosis [26]. The results of this study confirm and extend this evidence to show that FP contrasts the IL- 2 effects by decreasing Bcl-2 expression (fig. 4) and by restoring Bax (a Bcl-2 antagonist) expression (fig. 5) on PBTs from both asthmatic and normal subjects. The $\mathrm{Bax} / \mathrm{Bcl}-2$ ratio was further analysed because it appears to be an accurate determinant for apoptosis: lower $\mathrm{Bax} / \mathrm{Bcl}-2$ ratio promotes cell survival; whereas, higher $\mathrm{Bax} / \mathrm{Bcl}-2$ ratio promotes cell death [23]. Interestingly, FP significantly increased the $\mathrm{Bax} / \mathrm{Bcl}-2$ ratio on PBTs from asthmatics (table 1 ), suggesting that in activated T-lymphocytes, FP also promotes apoptosis by increasing the Bax expression, which in turn is able to counteract the antiapoptotic function of Bcl-2 by binding to this molecule.

Further evidence that FP exerts specific modulatory functions in activated T-cells, is its ability to generate a reduction in the number of Fas-positive cells in
PBTs from asthmatics. Accordingly, it has been demonstrated that the treatment of asthmatic patients with either oral or inhaled steroids decreases Fas and increases Fas-L expression in T-lymphocytes present in bronchial submucosa [27]. In this regard, it has been proposed that in asthma the increased expression of Fas-L in the epithelium of steroid-treated asthmatics may exert an additional cytotoxic activity toward Fas-bearing T-lymphocytes infiltrating the submucosa [27]. Moreover, this study demonstrated that FP significantly reduced CD25 on the surface of PBTs (fig. 7), suggesting that FP counteracts the IL-2 activity not only by inhibiting the production of this cytokine [28] but also by reducing the expression of its receptor. It is conceivable that the reduction of CD25 and Fas expression is also due to the ability of FP to induce apoptosis preferentially on activated PBTs, characterized by a greater expression of Fas and CD25 molecules. Accordingly, the treatment with FP reduces the number of CD3/HLA-DR positive T-lymphocytes in the PBTs of asthmatic patients [29]. Taken together, these findings support the hypothesis that FP reduces the presence of activated T-lymphocytes at the site of inflammation by promoting T-cell apoptosis. Moreover, the finding that IL-2stimulated PBTs are more responsive to the formerly described effects of FP than unstimulated PBTs (figs. 1, 4-7) might be explained by the presence of a higher expression of GR on activated PBTs. In this regard, 

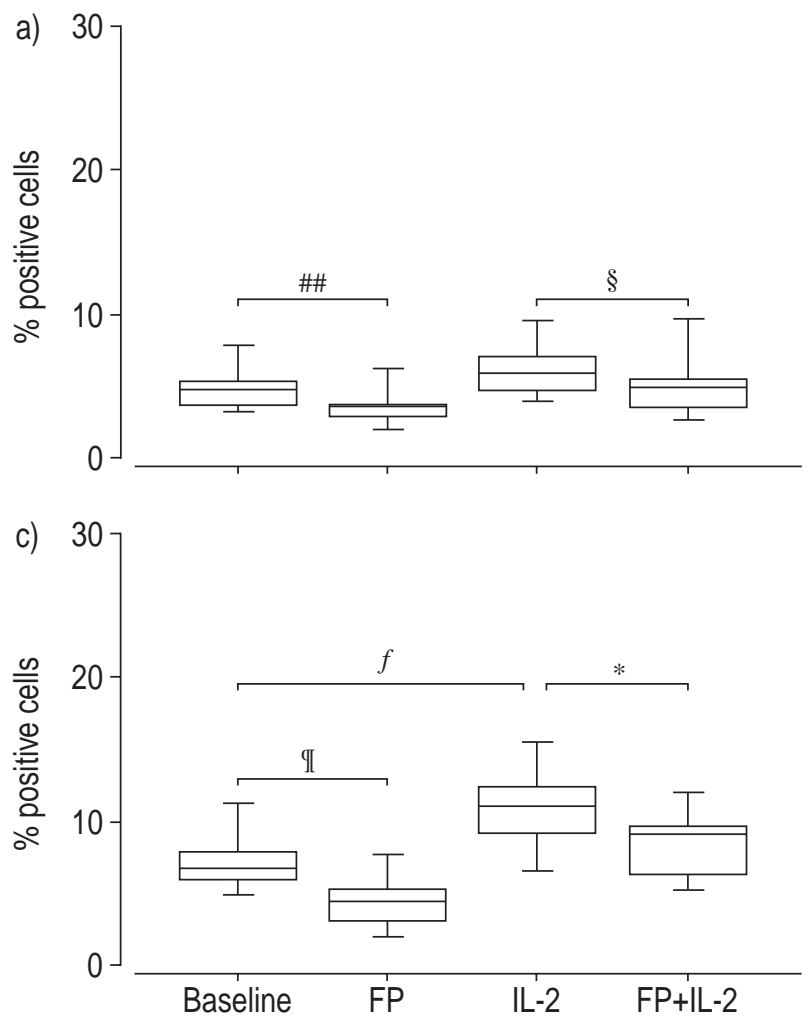

b)

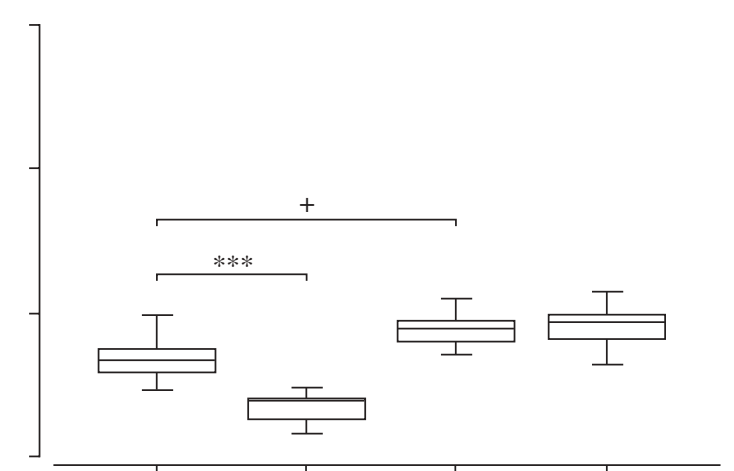

d)

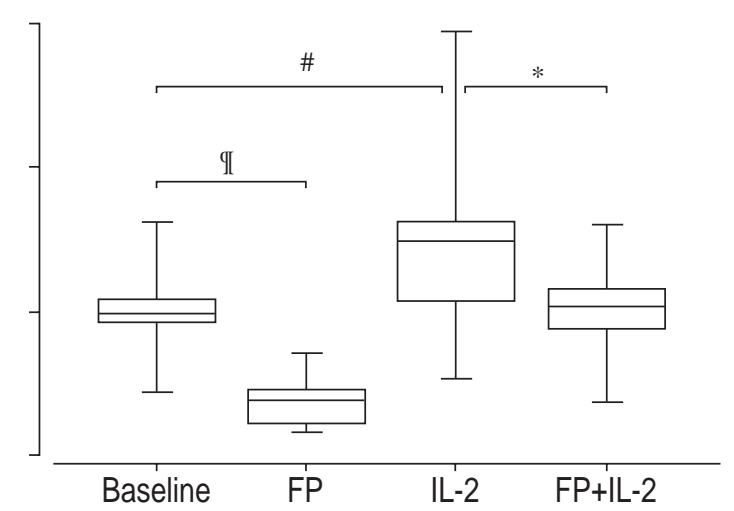

Fig. 7. - Expression of CD25 by peripheral blood T-lymphocytes (PBTs) isolated from normal (a: $72 \mathrm{~h}, \mathrm{~b}$ : $144 \mathrm{~h}$; $\mathrm{n}=10$ ) and asthmatic (c: $72 \mathrm{~h}, \mathrm{~d}$ : $144 \mathrm{~h} ; \mathrm{n}=10$ ) subjects. PBTs were isolated as described in "Materials and methods" and CD25 expression was evaluated by using an anti-CD25 monoclonal antibody on PBTs cultured in medium alone, in the presence of fluticasone propionate (FP), in the presence of interleukin-2 (IL-2), and in the presence of both FP and IL-2. Data are expressed as \% of positive cells (mean \pm SD). Medium $0 \mathrm{~h}$ : normals versus asthmatics $\mathrm{p}<0.04$; medium $72 \mathrm{~h}$ : normals versus asthmatics $\mathrm{p}<0.04$; medium $144 \mathrm{~h}$ : normals versus asthmatics $\mathrm{p}<0.006$. Statistical analysis was by analysis of variance (ANOVA) test. *: $\mathrm{p}<0.05 ;{ }^{\#}: \mathrm{p}<0.003 ;{ }^{\uparrow}: \mathrm{p}<0.03 ;{ }^{+}: \mathrm{p}<0.009 ;{ }^{\S}: \mathrm{p}<0.007 ;{ }^{f}$ : $\mathrm{p}<0.005$; $^{\# \#}: \mathrm{p}<0.002 ; * * *: \mathrm{p}<0.001$.

it has been demonstrated that phytohaemogluttininactivated PBTs express a higher number of GR messenger ribonucleic acid (mRNA) [25] than resting PBTs. Finally, no correlation between apoptosis and related markers (Bcl-2, Bax, and Fas; data not shown) was found, suggesting that FP-induced apoptosis is due to the concomitant activation of a variety of mechanisms, rather than the involvement of a single pathway. Moreover, in asthmatic patients, the discrepancy between the number of apoptotic cells and of apoptosis markers may also be related to an altered apoptotic potential of PBTs from asthmatic patients, as well as to the ongoing inflammatory process [30].

In conclusion, these findings suggest that fluticasone propionate is able to increase apoptosis and to modulate apoptosis related markers in interleukin2-stimulated peripheral blood T-lymphocytes and indicates an additional mechanism by which its antiinflammatory effects are exerted.

\section{References}

1. Vignola AM, Chanez P, Campbell AM, et al. Airway inflammation in mild intermittent and in persistent asthma. Am J Respir Crit Care Med 1998; 157: 403409.
2. Corrigan CJ, Hartnell A, Kay AB. T lymphocyte activation in acute severe asthma. Lancet 1988; 1: 1129-1132.

3. Robinson D, Hamid Q, Bentley A, Ying S, Kay AB, Durham SR. Activation of CD4+ T cells, increased TH2-type cytokine mRNA expression, and eosinophil recruitment in bronchoalveolar lavage after allergen inhalation challenge in patients with atopic asthma. J Allergy Clin Immunol 1993; 92: 313-324.

4. Vignola AM, Chanez P, Chiappara G, et al. Evaluation of apoptosis of eosinophils, macrophages, and $\mathrm{T}$ lymphocytes in mucosal biopsy specimens of patients with asthma and chronic bronchitis. J Allergy Clin Immunol 1999; 103: 563-573.

5. Corrigan CJ, Kay AB. CD4 T-lymphocyte activation in acute severe asthma. Relationship to disease severity and atopic status. Am Rev Respir Dis 1990; 141: 970-977.

6. Cohen JJ, Duke RC, Fadok VA, Sellins KS. Apoptosis and programmed cell death in immunity. Annu Rev Immunol 1992; 10: 267-293.

7. Lenardo MJ, Boehme S, Chen L, et al. Autocrine feedback death and the regulation of mature T lymphocyte antigen responses. Int Rev Immunol 1995; 13: 115-134.

8. Goodnow CC. Balancing immunity and tolerance: deleting and tuning lymphocyte repertoires. Proc Natl Acad Sci 1996; 93: 2264-2271.

9. Oltvai ZN, Milliman CL, Korsmeyer SJ. Bcl-2 
heterodimerizes in vivo with a conserved homolog, Bax, that accelerates programmed cell death. Cell 1993; 74: 609-619.

10. Alderson MR, Tough TW, Davis ST, et al. Fas ligand mediates activation-induced cell death in human $\mathrm{T}$ lymphocytes. J Exp Med 1995; 181: 71-77.

11. Van-Parijs L, Biuckians A, Abbas AK. Functional roles of Fas and Bcl-2-regulated apoptosis of $\mathrm{T}$ lymphocytes. J Immunol 1998; 160: 2065-2071.

12. Booth H, Gordiner PV, Ward C, Walls A, Hendrick DJ, Walters EM. Effect of fluticasone on airway inflammation as assessed by broncoalveolar lavage (BAL). Eur Respir J 1993; 6: Suppl. 17, 584s.

13. Hagan JB, Kita H, Gleich GJ. Inhibition of interleukin-5 mediated eosinophil viability by fluticasone 17-propionate: comparison with other glucocorticoids. Clin Exp Allergy 1998; 28: 999-1006.

14. Aas K. Heterogeneity of bronchial asthma. Allergy 1981; 36: 3-10.

15. Cruikshank WW, Long A, Tarpy RE, et al. Early identification of interleukin-16 (lymphocyte chemoattractant factor) and macrophage inflammatory protein 1 alpha (MIP1 alpha) in bronchoalveolar lavage fluid of antigen-challenged asthmatics. Am J Respir Cell Mol Biol 1995; 13: 738-747.

16. Johnson M. Development of fluticasone propionate and comparison with other inhaled corticosteroids. J Allergy Clin Immunol 1998; 101: S434-S439.

17. Bouffard DY, Momparler RL. Comparison of the induction of apoptosis in human leukemic cell lines by $2^{\prime}, 2^{\prime}$-difluorodeoxycytidine (gemcitabine) and cytosine arabinoside. Leuk Res 1995; 19: 849-856.

18. Mor F, Cohen IR. IL-2 rescues antigen-specific T cells from radiation or dexamethasone-induced apoptosis. Correlation with induction of Bcl-2. J Immunol 1996; 156: 515-522.

19. Nieto MA, Lopez RA. IL-2 protects T lymphocytes from glucocorticoid-induced DNA fragmentation and cell death. J Immunol 1989; 143: 4166-4170.

20. Fokkens WJ, van dMJ, Braat JP, Overbeek SE, Hooijkaas $\mathrm{H}$. The effect of intranasal and inhaled corticosteroids in healthy volunteers on the number of circulating lymphocytes and lymphocyte subsets. Allergy 1999; 54: 158-164.

21. Haslett C, Savill JS, Whyte MK, Stern M, Dransfield I, Meagher LC. Granulocyte apoptosis and the control of inflammation. Phil Trans $R$ Soc Lond $B$ Biol Sci 1994; 345: 327-333.

22. White E. Life, death, and the pursuit of apoptosis. Genes Dev 1996; 10: 1-15.

23. Tuosto L, Cundari E, Montani MS, Piccolella E. Analysis of susceptibility of mature human T lymphocytes to dexamethasone-induced apoptosis. Eur J Immunol 1994; 24: 1061-1065.

24. Brunetti M, Martelli N, Colasante A, Piantelli M, Musiani P, Aiello FB. Spontaneous and glucocorticoidinduced apoptosis in human mature $\mathrm{T}$ lymphocytes. Blood 1995; 86: 4199-4205.

25. Lanza L, Scudeletti M, Puppo F, et al. Prednisone increases apoptosis in in vitro activated human peripheral blood T lymphocytes. Clin Exp Immunol 1996; 103: 482-490.

26. Akbar AN, Salmon M. Cellular environments and apoptosis: tissue microenvironments control activated T-cell death. Immunol Today 1997; 18: 72-76.

27. Druilhe A, Wallaert B, Tsicopoulos A, et al. Apoptosis, proliferation, and expression of $\mathrm{Bcl}-2$, Fas, and Fas ligand in bronchial biopsies from asthmatics. $\mathrm{Am}$ J Respir Cell Mol Biol 1998; 19: 747-757.

28. Diaz-Sanchez D, Tsien A, Fleming J, Saxon A. Effect of topical fluticasone propionate on the mucosal allergic response induced by ragweed allergen and diesel exhaust particle challenge. Clin Immunol 1999; 90: 313-322.

29. Bootsma GP, Koenderman L, Dekhuijzen PN, Festen J, Lammers JW, Van Herwaarden CL. FasL expression on epithelial cells: the Bottazzo-Feldman hypothesis revisited. Allergy 1998; 53: 653-661.

30. Jayaraman S, Castro M, O'Sullivan M, Bragdon MJ, Holtzman MJ. Resistance to Fas-mediated T cell apoptosis in asthma. J Immunol 1999; 162: 17171722. 